\title{
Performance Analysis of Coded $M$-ary Orthogonal Signaling Using Errors-and-Erasures Decoding Over Frequency-Selective Fading Channels
}

\author{
Lie-Liang Yang, Member, IEEE, and Lajos Hanzo, Senior Member, IEEE
}

\begin{abstract}
The performance of $M$-ary orthogonal signaling schemes employing Reed-Solomon (RS) codes and redundant residue number system (RRNS) codes is investigated over frequency-selective Rayleigh fading channels. "Errors-and-erasures" decoding is considered, where erasures are judged based on two low-complexity, low-delay erasure insertion schemes-Viterbi's ratio threshold test (RTT) and the proposed output threshold test (OTT). The probability density functions (PDF) of the ratio associated with the RTT and that of the demodulation output in the OTT conditioned on both the correct detection and erroneous detection of $M$-ary signals are derived, and the characteristics of the RTT and OTT are investigated. Furthermore, expressions are derived for computing the codeword decoding error probability of RS codes or RRNS codes based on the above PDFs. The OTT technique is compared to Viterbi's RTT, and both of these are compared to receivers using "error-correction only" decoding over frequency-selective Rayleigh-fading channels. The numerical results show that by using "errors-and-erasures" decoding, RS or RRNS codes of a given code rate can achieve higher coding gain than that without erasure information, and that the OTT technique outperforms the RTT, provided that both schemes are operated at the optimum decision thresholds.
\end{abstract}

Index Terms-“Errors-and-erasures" decoding, $M$-ary orthogonal signaling, Rayleigh fading, redundant residue number system codes, Reed-Solomon codes.

\section{INTRODUCTION}

$\mathbf{F}$ ORWARD error-correction (FEC) is often used for mitigating the effects of fading over wireless communication channels. For most so-called "errors-and-erasures" decoding schemes [1], erasures are preferable to error correction, since typically more erasures than errors can be corrected. Hence, it is advantageous to determine the reliability of the received symbols and erase the low-reliability symbols prior to the decoding process. There are a number of methods for generating reliability-based side information and their performance has been analyzed, for example, in [2]-[9].

In this paper, we consider the properties of the ratio threshold test (RTT), which was originally suggested by Viterbi [3], and those of our proposed output threshold test (OTT), which is defined during our further discourse. Both of them are then invoked in the context of $M$-ary orthogonal signaling, in order

Manuscript received June 22, 2000; revised September 4, 2000. This work was supported by EPSRC, U.K. and the Commission of the European Communities, Brussels, Belgium.

The authors are with the Department of Electronics and Computer Science, University of Southampton, SO17 1BJ, U.K. (e-mail: 1h@ecs.soton.ac.uk).

Publisher Item Identifier S 0733-8716(01)00128-7. to generate channel-quality related information and-ultimately - to enhance the performance of $M$-ary orthogonal modulated systems. Viterbi's RTT [3] was originally proposed for mitigating the partial-band interference or multitone interference, and when invoked as an erasure insertion scheme, it is suboptimum, as discussed in [5] and [6]. However, it is practically the simplest erasure insertion scheme, which is robust to almost all channel impairments. Kim and Stark [7] have invoked it for mitigating the effect of Rayleigh-fading and have analyzed the performance limits of Reed-Solomon (RS) codes using "errors-and-erasures" decoding. The contribution of this paper is to investigate the performance of both RS codes [10] and that of the novel class of redundant residue number system (RRNS) codes [18], [19], when $M$-ary orthogonal signaling is employed in conjunction with RTT- or OTT-based detection over frequency-selective Rayleigh-fading channels. In contrast to the previously published simulation-based performance evaluation [5], [6] or to analyzing the performance limits [7], in this paper we derive the exact probability density functions (PDF) of both the RTT and the OTT at the demodulator's output conditioned on both the correct detection and erroneous detection of the $M$-ary signals. These PDFs are then used to investigate the characteristics of the RTT and OTT over the above-mentioned Rayleigh-fading channels, and also to derive the expressions of the codeword decoding error probability. The performance of the associated $M$-ary orthogonal signaling schemes in conjunction with RS codes and RRNS codes using "errors-and-erasures" decoding employing the RTT or OTT is then estimated and compared to that using "error-correction-only" decoding without side information. We also estimate and compare the optimum code rate for RS codes upon employing different decoding schemes and different diversity capability arrangements.

RS codes [10] constitute a maximum-minimum distance class of codes, having the capability of correcting/detecting symbol errors. By contrast, the RRNS codes are less widely known in the field of wireless communications. The so-called residue number system (RNS) arithmetic has drawn wide attention in computer science and in high-speed parallel signal processing due to its fault-tolerant properties [11], [12]. Error control coding theory using RRNS codes has recently been substantially advanced [13]-[18], raising our interest in investigating the properties of RRNS codes in different application environments, while extending its applications to new fields. Furthermore, the RNS arithmetic and the RRNS codes have 
been proposed by the authors for parallel data transmission and protection in wireless communications [19], [20].

An RNS is defined [11] by the choice of $k$ positive integers $m_{i},(i=1,2, \ldots, k)$ referred to as moduli. If all the moduli are pairwise relative primes, any integer $N$, describing the information symbols to be transmitted in this paper, can be uniquely and unambiguously represented by the residue sequence $\left(r_{1}, r_{2}, \ldots, r_{k}\right)$ in the range $0 \leq N<M_{I}$ where $r_{i}=N\left(\bmod m_{i}\right)$ represents the residue digits of $N$ upon division by $m_{i}$, and $M_{I}=\prod_{i=1}^{k} m_{i}$ is the information dynamic range, i.e., the legitimate range of the information symbols $N$. According to the Chinese reminder theorem (CRT) [16], for any given $k$-tuple $\left(r_{1}, r_{2}, \ldots, r_{k}\right)$, where $0 \leq r_{i}<m_{i}$, there exists one and only one integer $N$ such that $0 \leq N<M_{I}$ and $r_{i}=N\left(\bmod m_{i}\right)$, which allows us to uniquely recover the message $N$ from the received residue digits.

For incorporating error control [16], [18], the RNS has to be designed with redundant moduli, which is referred to as an RRNS code. An RRNS code is obtained by appending an additional $(n-k)$ number of moduli $m_{k+1}, m_{k+2}, \ldots, m_{n}$, to the previously introduced RNS, in order to form an RRNS code of $n$ positive, pairwise relative prime moduli. The redundant moduli have to obey $m_{k+j} \geq \max \left\{m_{1}, m_{2}, \ldots, m_{k}\right\}$. Now an integer $N$ in the range $\left[0, M_{I}\right)$ is represented as an $n$-tuple residue sequence, $\left(r_{1}, r_{2}, \ldots, r_{n}\right)$ with respect to the $n$ moduli, and consequently forms an $\operatorname{RRNS}(n, k)$ code.

RRNS and RS codes exhibit similar coding properties [14]-[16], [18], both being maximum distance separable codes. An $\operatorname{RRNS}(n, k)$ code, where the information dynamic range is $\left[0, \prod_{i=1}^{k} m_{i}\right)$ and the total code dynamic range is $\left[0, \prod_{i=1}^{n} m_{i}\right)$, has a minimum distance of $(n-k+1)$, and hence it is capable of detecting $(n-k)$ or less residue digit errors and correct up to $\lfloor(n-k) / 2\rfloor$ random residue digit errors, where $\lfloor x\rfloor$ represents the largest integer not exceeding $x$. An $\operatorname{RRNS}(n, k)$ code is capable of correcting $t$ random residue digit errors and simultaneously correcting $\beta$ residue erasures, if and only if $2 t+\beta \leq(n-k)$ [18]. A further property of RRNS codes is that the residue digits based on different moduli can be processed independently of each other. Hence, as indicated in [18], an $\operatorname{RRNS}(n, k)$ code reduces to an $\operatorname{RRNS}(n-\beta, k)$ code after $\beta$ out of the $n$ residue digits are erased. The erased symbols are not required to be considered during the decoding of RRNS codes, and hence the "errors-and-erasures" decoding of RRNS codes can be simplified to "error-correction-only" decoding of the reduced RRNS codes, consequently simplifying the decoding process.

RS codes and RRNS codes exhibit similar distance properties, and hence they are capable of achieving a similar coding performance for identical-length RS and RRNS codes. However, the RS code symbols possess a constant base of $2^{m}$ for $m$-bit symbols, while RRNS code symbols are related to a group of bases from the set of moduli $\left\{m_{1}, m_{2}, \ldots, m_{n}\right\}$. The length $n$ of RS codes is usually related to the legitimate range of code symbols given by $n=2^{m}-1$, and short RS codes having a high symbol dynamic range can only be obtained by shortening long RS codes. However, the decoding complexity of the shortened RS codes is typically similar to that of the original codes [1], [10]. By contrast, it is relatively straightforward to design arbitrary-length RRNS codes having a large variety of symbol dynamic ranges.

The remainder of this paper is organized as follows. In Section II, we introduce the erasure insertion test and the associated expression of the codeword decoding error probability. In Section III, we derive the conditional PDFs of the RTT and analyze their characteristics, while Section IV investigates the OTT and its characteristics. The associated performance results of the $M$-ary orthogonal signaling schemes invoking RTT and OTT are provided in Section V. Finally, in Section VI we present our conclusions.

\section{ERASURE INSERTION TEST}

Our initial discussions in this section are related to RS codes and RRNS codes. The signaling scheme to be used from Section III onwards is the $M$-ary orthogonal scheme [24] employing the transmitter and receiver schematics of Fig. 1, which will be described during our later discussions.

Let $H_{1}$ and $H_{0}$ represent the hypotheses that a received symbol is demodulated correctly and erroneously, respectively, according to a given optimum detection criterion, such as the maximum a posteriori probability (MAP), maximum likelihood (ML), or minimum error probability, etc. We refer to this detection of data as the 1st stage decision, as indicated in Fig. 1. Let us denote the variable subjected to an erasure insertion decision by $\boldsymbol{Y}$. Given that $H_{i}(i=0,1)$ was stipulated, $\boldsymbol{Y}$ has a conditional density of $f\left(\boldsymbol{y} \mid H_{i}\right)$. Then, the erasure insertion strategy can be formulated as a second-stage decision concerning erasure insertion, in order to distinguish between the following hypotheses:

$$
\begin{aligned}
& H_{0} \text { : Erroneous demodulated symbol: } \\
& \quad \text { insert an erasure. } \\
& H_{1} \text { : Correct demodulated symbol: } \\
& \quad \text { output an RS or RRNS code symbol. }
\end{aligned}
$$

Let the observation space be denoted by $\mathcal{R}$, and assume that $\mathcal{R}_{0}$ and $\mathcal{R}_{1}$ are the sets of values in $\mathcal{R}$ that map into the decisions $H_{0}$ and $H_{1}$, respectively, where $\mathcal{R}_{0} \cup \mathcal{R}_{1}=\mathcal{R}$. Let $P_{c}, P_{t}$, and $P_{e}$ represent the correct symbol probability, symbol error probability, and symbol erasure probability, respectively, after the 2 nd stage decision of Fig. 1 but before FEC decoding. Then these probabilities can be expressed as

$$
\begin{aligned}
& P_{c}=P\left(H_{1}\right) \int_{\mathcal{R}_{1}} f\left(\boldsymbol{y} \mid H_{1}\right) d y \\
& P_{t}=P\left(H_{0}\right) \int_{\mathcal{R}_{1}} f\left(\boldsymbol{y} \mid H_{0}\right) d y \\
& P_{e}=P\left(H_{0}\right) \int_{\mathcal{R}_{0}} f\left(\boldsymbol{y} \mid H_{0}\right) d y+P\left(H_{1}\right) \int_{\mathcal{R}_{0}} f\left(\boldsymbol{y} \mid H_{1}\right) d y
\end{aligned}
$$

which obey the relationship of

$$
P_{e}=1-P_{c}-P_{t}
$$

According to (3), the erasure probability is constituted by two terms. The first term is based on the hypothesis of $H_{0}$, 


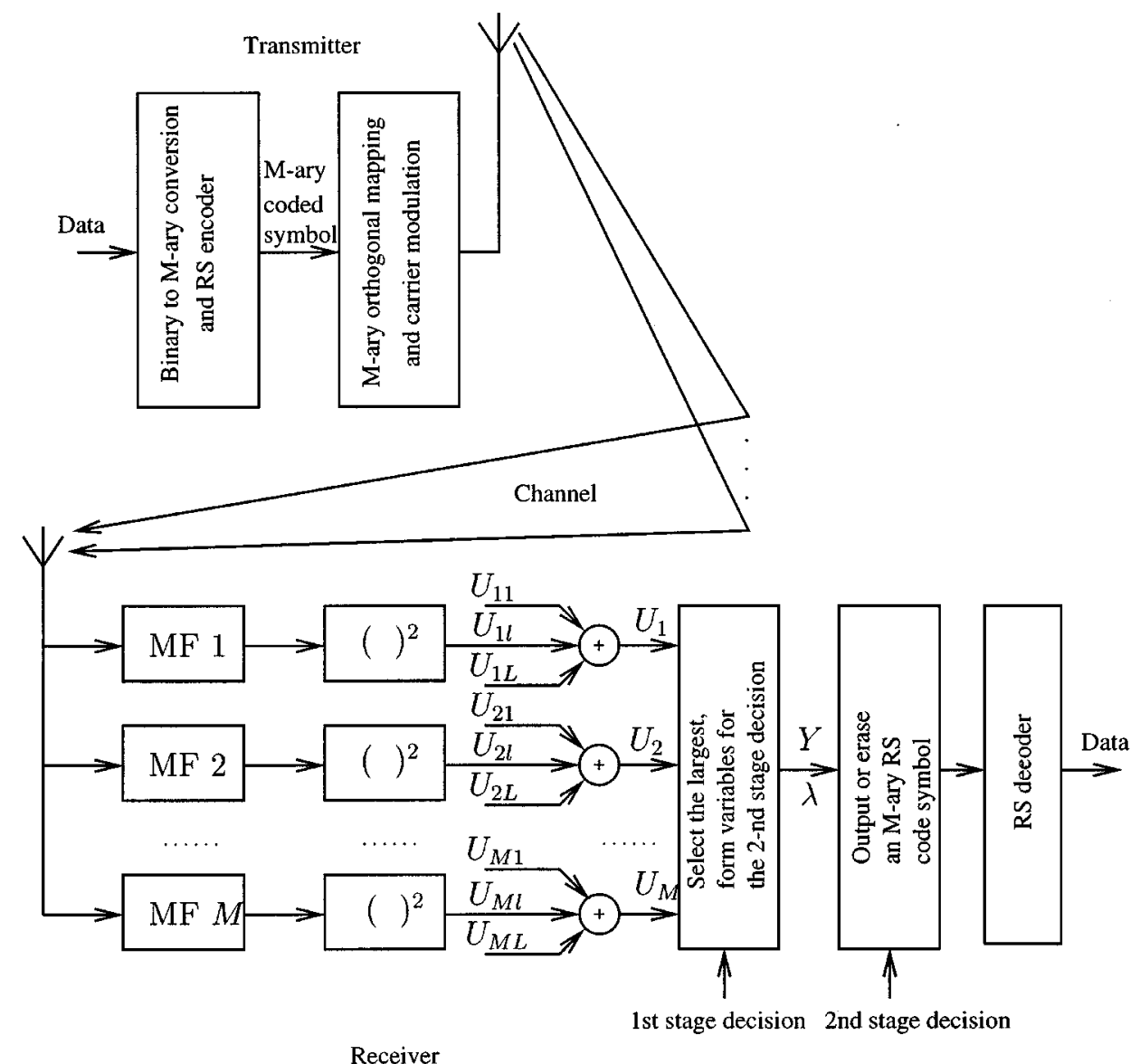

Fig. 1. Transmitter and receiver schematic of an $M$-ary orthogonal signaling scheme using square-law detection, equal gain combining, first- and second-stage decisions as well as RS or RRNS channel coding.

i.e., when a symbol was detected erroneously and hence erasure is required, while the second term accrues from the unintentional erasure of a symbol, which was detected correctly, due to its mapping into $\mathcal{R}_{0}$. Consequently, in order to minimize the decoding error probability for RS codes or RRNS codes using "errors-and-erasures" decoding, the optimum erasure insertion strategy to minimize the codeword decoding error probability is that of maximizing the erasure probability under the hypothesis $H_{0}$ - which corresponds to the first term of (3) - and, simultaneously, minimizing the erasure probability under the hypothesis $H_{1}$, which corresponds to the second term of (3). Then the erroneously demodulated symbols should be erased with the highest probability, while the correctly demodulated symbols should be erased with the lowest probability.

Let $\left\{U_{1}, U_{2}, \ldots, U_{M}\right\}$ be the decision variables of an $M$-ary orthogonal signaling scheme in the context of making a decision, as to whether output an $M$-ary symbol or to insert an erasure. Specially, if all decision variables $\boldsymbol{Y}=\left(U_{1}, U_{2}, \ldots, U_{M}\right)$ are considered, the erasure insertion scheme is optimum in the sense of minimizing Bayes's risk [5], [6]. In Viterbi's RTT, the decision variable subjected to an erasure insertion was defined as [3]

$$
\boldsymbol{Y}=\lambda=\frac{Y_{1}={ }^{1} \max \left\{U_{1}, U_{2}, \ldots, U_{M}\right\}}{Y_{2}={ }^{2} \max \left\{U_{1}, U_{2}, \ldots, U_{M}\right\}}
$$

where $Y_{1}={ }^{1} \max \{\cdot\}$ and $Y_{2}={ }^{2} \max \{\cdot\}$ represent the maximum and the "second maximum" of the decision variables of $\left\{U_{1}, U_{2}, \ldots, U_{M}\right\}$, respectively. If only $\lambda$ is observed, this erasure decision scheme is also optimum. However, in the RTT, only the maximum and the "second maximum" of the decision variables $\left\{U_{1}, U_{2}, \ldots, U_{M}\right\}$ are employed for making an erasure insertion decision; hence the RTT is not optimum. By contrast, in our proposed OTT, the decision variable subjected to an erasure insertion is defined as

$$
Y=Y=\max \left\{U_{1}, U_{2}, \ldots, U_{M}\right\}
$$

where only the maximum, i.e., the actual demodulator output is observed. Hence, the OTT is not the optimum decision metric either. However, our numerical results in Section V show that the OTT outperforms the RTT over the dispersive fading channels considered, which is likely to be a consequence of the fact that the associated PDFs become less separable after the division of the maximum by the "second maximum." This is because the performance of an erasure insertion scheme depends on the separability of the PDFs under different hypotheses $\left(H_{0}\right.$ and $\left.H_{1}\right)$. We note here that the OTT might not be suitable for channels having partial or multitone interference, where the partial or multitone interference may result in an erroneous output associated with a high amplitude. By contrast, due to the similar effects 
of partial or multitone interference on all correlation branches of the $M$-ary orthogonal demodulator [3], Viterbi's ratio defined in (5) is less prone to erroneous decisions, when subjected to interference. Due to the associated advantages and disadvantages of the RTT and OTT, in this paper these metrics are studied comparatively. However, since the optimum Bayesian erasure insertion approach is analytically intractable [5], [6], where all decision variables $\left\{U_{1}, U_{2}, \ldots, U_{M}\right\}$ are considered, the analysis of this particular case is not considered in this paper.

The decoding performance of RS codes or RRNS codes can be quantified in terms of the codeword decoding error probability, $P_{E}$, which can be computed as follows. Let $\omega_{i}, i=$ $1,2, \ldots, n$ be

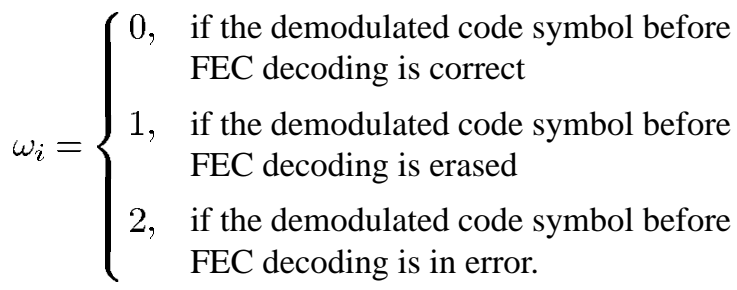

According to the coding theory of RS [1] and RRNS codes [14], [15], an $(n, k)$ RS or RRNS code can correct any set of $t$ symbol errors and $\beta$ symbol erasures provided that $2 t+\beta \leq(n-k)$. Hence, with the aid of (7), it can be shown that a codeword can be correctly decoded using "errors-and-erasures" decoding, if the sum of $\omega_{1}, \omega_{2}, \ldots, \omega_{n}$ corresponding to the $n$ code symbols of the codeword, i.e., $\sum_{i=1}^{n} \omega_{i}$, does not exceed $(n-k)$. Consequently, the probability $P_{E}$ of codeword decoding errors using "errors-and-erasures" decoding can be expressed as

$$
P_{E}=\sum_{j=n-k+1}^{n} P\left(\sum_{i=1}^{n} \omega_{i}=j\right) .
$$

If we assume that the positions of symbol errors and symbol erasures within a codeword are independent, for example, due to sufficiently long interleaving, then the codeword decoding error probability, $P_{E}$ can be expressed in the form of [6]

$$
P_{E}=\sum_{i=0}^{n} \sum_{j=j_{0}(i)}^{n-i}\left(\begin{array}{c}
n \\
i
\end{array}\right)\left(\begin{array}{c}
n-i \\
j
\end{array}\right) P_{t}^{i} P_{e}^{j}\left(1-P_{t}-P_{e}\right)^{n-i-j}
$$

where $j_{0}(i)=\max \{0, n-k+1-2 i\}$, and $P_{t}$ and $P_{e}$ represent the symbol error probability and symbol erasure probability before channel decoding, respectively, which are given by (2) and (3). Equation (9) lends itself to the computation of the codeword decoding error probability, if the code is not excessively long. However, if the RS or the RRNSs codewords are long, the well-known Gaussian approximation [7] can be invoked, in order to simplify the computation, since under the assumption that the symbol errors and erasures are independent, the term $\sum_{i=1}^{n} \omega_{i}$ approaches a Gaussian distribution according to the central limit theorem, whose mean value is given by $M_{c}=E\left[\sum_{i=1}^{n} \omega_{i}\right]=n\left(P_{e}+2 P_{t}\right)$ [7], and variance by
$\sigma_{c}^{2}=\operatorname{Var}\left[\sum_{i=1}^{n} \omega_{i}\right]=n\left[1-P_{e}-\left(1-P_{e}-2 P_{t}\right)^{2}\right]$ [7]. Consequently, the codeword decoding error probability in (8) can be approximated as

$$
\begin{aligned}
P_{E} & =\int_{n-k+1}^{n} \frac{1}{\sqrt{2 \pi} \sigma_{c}} \exp \left(-\frac{\left(t-M_{c}\right)^{2}}{\sigma_{c}^{2}}\right) d t \\
& =Q\left(\eta_{1}\right)-Q\left(\eta_{2}\right)
\end{aligned}
$$

where $Q(x)$ is the Gaussian $Q$-function, which is defined as $Q(x)=(1 / \sqrt{2 \pi}) \int_{x}^{\infty} \exp \left(-\left(t^{2} / 2\right)\right) d t$, and

$$
\begin{aligned}
\eta_{1} & \approx \frac{\sqrt{n}\left(1-R_{c}-P_{e}-2 P_{t}\right)}{\sqrt{1-P_{e}-\left(1-P_{e}-2 P_{t}\right)^{2}}} \\
\eta_{2} & \approx \frac{\sqrt{n}\left(1-P_{e}-2 P_{t}\right)}{\sqrt{1-P_{e}-\left(1-P_{e}-2 P_{t}\right)^{2}}}
\end{aligned}
$$

where $R_{c}=k / n$ is the code rate.

Note that since RRNS codes use different bases or moduli for the different symbols' representation, the probabilities of $P_{c}, P_{t}$, and $P_{e}$ for different symbols in a codeword are different after the above-discussed two-stage decisions. Hence, the equations developed in this section can only be used for approximating the performance of RRNS codes using moduli, which are close to each other. Fortunately, in practice, the values of the relative prime moduli in RRNS codes are usually indeed selected to be as close as possible. Hence, if the RRNS code is not excessively long, satisfactory approximations using RS code based equations can usually be achieved. Consequently, in our analysis, we will not distinguish between these codes, noting that the equations constitute an approximation for RRNS codes.

Above we have developed the erasure insertion theory for the "errors-and-erasures" decoding of RS codes or RRNS codes. Nonbinary RS and RRNS code symbols are amenable to transmission using $M$-ary orthogonal signaling schemes. For example, an $M$-ary orthogonal signaling scheme using $M=64$, i.e., 6-bit symbols, has been proposed for the reverse link of IS-95 [23]. Let us now focus on studying the performance of the $M$-ary orthogonal signaling scheme employing RS or RRNS codes and using "errors-and-erasures" decoding.

\section{FURTHER STUdY OF VITERBI's RTT ERASURE INSERTION SCHEME OVER A RAYLEIGH FADING CHANNEL}

Viterbi's RTT has been widely studied in different fadingand interference-impaired environments [3]-[9] using both analysis and simulations. In this section, we develop an analytical framework for invoking the RTT over frequency-selective fading channels by deriving the PDFs of the RTT under the hypotheses of $H_{1}$ and $H_{0}$. With the aid of these PDFs, we then analyze the distribution characteristics of the RTT and derive the decoding error probability of RS codes or RRNS codes.

Consider the wireless communication system of Fig. 1 using $M$-ary orthogonal signaling over an independently and slowly fading Rayleigh channel, having $L$ independent, identically distributed (iid) multipath components. Each signaling waveform in the symbol interval $[0, T)$ is equiproabable and contains the same energy $\xi$. The received signal is corrupted by additive white Gaussian noise (AWGN) having double-sided power spectral density of $N_{0} / 2$. The noise associated with 
each diversity component is assumed to be iid. The optimum receiver for each diversity branch is a matched-filter followed by a square-law envelope detector [22] as shown in Fig. 1.

Let $U_{i l}, i=1,2, \ldots, M, l=1,2, \ldots, L$ be the output of the square-law envelope detector for the $i$ th symbol on the $l$ th diversity channel. Assume that the first element of the symbol alphabet is sent. Then, the decision variables $\left(U_{1}, U_{2}, \ldots, U_{M}\right)$ after equal gain combining (EGC) can be expressed as $[24, \mathrm{p}$. 788]

$$
\begin{aligned}
U_{1} & =\sum_{l=1}^{L}\left|2 \xi \alpha_{l} e^{-j \varphi_{l}}+N_{l 1}\right|^{2} \\
U_{i} & =\sum_{l=1}^{L}\left|N_{l i}\right|^{2}, \quad i=2,3, \ldots, M
\end{aligned}
$$

where $N_{l i}$ is a complex zero-mean Gaussian random variable with variance $4 \xi N_{0}$, and $\alpha_{l} e^{-j \varphi_{l}}$ represents the complex channel coefficient. Since we have modeled the channel's multipath components by independent slow Rayleigh fading, the channel coefficient $\alpha_{l} e^{-j \varphi_{l}}$ is also a complex zero-mean Gaussian random variable with variance $E\left[\alpha^{2}\right]$, where $E[\cdot]$ represents the expected value of the argument. Consequently, the PDFs of the decision variables $U_{1}$ and $U_{i}, i=2,3, \ldots, M$ are chi-square distributed with $2 L$ degree of freedom [24, p. 784]. After normalization by $4 \xi N_{0}$, the PDFs of $U_{1}$ and $U_{i}, i=2,3, \ldots, M$ can be expressed upon modifying Proakis' approach $[24$, p. 784, (14-4-31) and (14-4-32)] as

$$
\begin{aligned}
f_{U_{1}}(x) & =\frac{1}{\left(1+\bar{\gamma}_{0}\right)^{L}(L-1) !} x^{L-1} \exp \left(-\frac{x}{1+\bar{\gamma}_{0}}\right) \\
f_{U_{i}}(x) & =\frac{1}{(L-1) !} x^{L-1} \exp (-x) \\
& x \geq 0, i=2,3, \ldots, M
\end{aligned}
$$

where $\bar{\gamma}_{0}=\xi E\left[\alpha_{l}^{2}\right] / N_{0}=\xi E\left[\alpha^{2}\right] / N_{0}$ is the average signal-tonoise (SNR) ratio per diversity channel. The probability of error after maximum likelihood detection (MLD), i.e., the a priori probability of the erroneous decision hypothesis $H_{0}$, as we discussed it in Section II, is given by [24, p. 789, (14-4-44)], which is expressed as

$$
\begin{aligned}
P\left(H_{0}\right)= & -\int_{0}^{\infty} \frac{1}{\left(1+\bar{\gamma}_{0}\right)^{L}(L-1) !} y^{L-1} \exp \left(-\frac{y}{1+\bar{\gamma}_{0}}\right) \\
& \cdot\left[1-\exp (-y) \sum_{k=0}^{L-1} \frac{y^{k}}{k !}\right]^{M-1} d y .
\end{aligned}
$$

The a priori probability of the correct decision hypothesis $H_{1}$ is given by

$$
P\left(H_{1}\right)=1-P\left(H_{0}\right) \text {. }
$$

Viterbi's RTT was defined in (5). The PDFs of the maximum and the "second maximum" of ${ }^{1} \max \{\cdot\}$ and ${ }^{2} \max \{\cdot\}$ under the correct detection hypothesis of $H_{1}$ and under the erroneous detection hypothesis of $H_{0}$, as well as the corresponding PDFs of the RTT defined in (5) are derived in the Appendix. The PDFs of the RTT under the hypotheses of $H_{1}$ and $H_{0}$ are expressed as

$$
\begin{aligned}
f_{\lambda}\left(y \mid H_{1}\right) & \frac{1}{\left[P\left(H_{1}\right)\right]^{2} P\left(Y_{2}<Y_{1} \mid H_{1}\right)} \frac{M-1}{\left(1+\bar{\gamma}_{0}\right)^{L}[(L-1) !]^{2}} y^{L-1} \\
& \cdot \int_{0}^{\infty} x^{2 L-1} \exp \left(-x-\frac{x y}{1+\bar{\gamma}_{0}}\right) \\
& \cdot[1-\Psi(x y)]^{M-1}[1-\Psi(x)]^{M-2} \Psi\left(\frac{x}{1+\bar{\gamma}_{0}}\right) d x \\
f_{\lambda}\left(y \mid H_{0}\right) & y \geq 1 \\
= & \frac{1}{\left[P\left(H_{0}\right)\right]^{2} P\left(Y_{2}<Y_{1} \mid H_{0}\right)} \frac{(M-1)^{2}}{[(L-1) !]^{2}} y^{L-1} \\
& \cdot \int_{0}^{\infty} x^{2 L-1} \exp (-x y)[1-\Psi(x y)]^{M-2} \\
& \cdot\left[1-\Psi\left(\frac{x y}{1+\bar{\gamma}_{0}}\right)\right] \Psi(x)[1-\Psi(x)]^{M-3} \\
& \cdot\left\{\frac{1}{\left(1+\bar{\gamma}_{0}\right)^{L}} \exp \left(-\frac{x}{1+\bar{\gamma}_{0}}\right)[1-\Psi(x)]\right. \\
& \left.+(M-2) \exp (-x)\left[1-\Psi\left(\frac{x}{1+\bar{\gamma}_{0}}\right)\right]\right\} d x \\
& y \geq 1
\end{aligned}
$$

where the short-hand $\Psi(t)$ was defined as

$$
\Psi(t)=\exp (-t) \sum_{k=0}^{L-1} \frac{t^{k}}{k !}
$$

and $P\left(Y_{2}<Y_{1} \mid H_{i}\right), i=0,1$ is the probability of $Y_{2}<Y_{1}$ conditioned on the hypothesis $H_{i}$, which is expressed as

$P\left(Y_{2}<Y_{1} \mid H_{i}\right)=\int_{0}^{\infty}\left[\int_{0}^{y_{1}} f_{Y_{2}}\left(y_{2} \mid H_{i}\right) d y_{2}\right] f_{Y_{1}}\left(y_{1} \mid H_{i}\right) d y_{1}$

where $f_{Y_{2}}\left(y_{2} \mid H_{i}\right)$ and $f_{Y_{1}}\left(y_{1} \mid H_{i}\right)$ were given by (34) and (32) or (39) and (37) for the hypotheses $H_{1}$ or $H_{0}$, respectively. Note that for RRNS codes rather than RS codes, $M$ in the above equations should be replaced by the average value of the moduli used, as we discussed previously.

The properties of Viterbi's RTT can be studied with the aid of the PDFs of $f_{\lambda}\left(y \mid H_{1}\right)$ and $f_{\lambda}\left(y \mid H_{0}\right)$ shown in Figs. 2 and 3 for a range of parameters, which are summarized in the figures. During our elaborations, we used the signal-to-noise ratio per bit, which was obtained by computing $\gamma_{b}=L \bar{\gamma}_{0} / \log _{2} M$, where $\log _{2} M$ is the number of bits per symbol. From the curves we observe that for a given value of $M$, the peak of the distribution of $f_{\lambda}\left(y \mid H_{1}\right)$ will shift to the right-hand side, while that of $f_{\lambda}\left(y \mid H_{0}\right)$ is around $\lambda \approx 1$. Furthermore, we observe in Fig. 3 that the peak of the distribution of $f_{\lambda}\left(y \mid H_{0}\right)$ becomes lower, as the SNR per bit increases. However, we infer from Fig. 2 that for a given value of $\gamma_{b}$, the peaks of the distributions of $f_{\lambda}\left(y \mid H_{0}\right)$ 


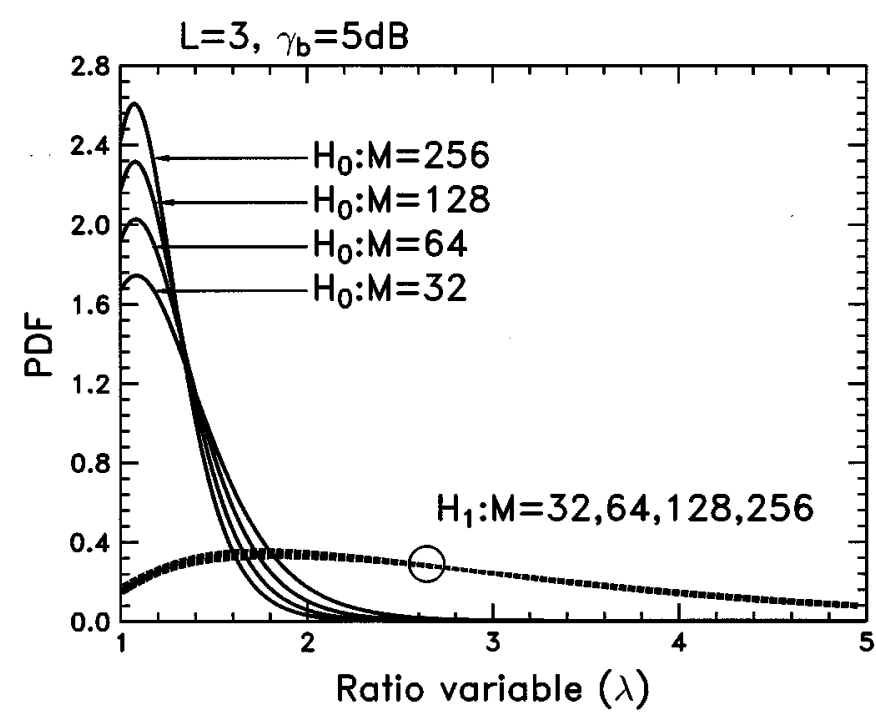

Fig. 2. RTT: The PDF of $\lambda={ }^{1} \max \{\cdot\}^{2} \max \{\cdot\}$ according to (19) and (20) under the hypotheses of $H_{1}$ and $H_{0}$ using $M=32,64,128,256$, diversity order of $L=3$, signal-to-noise per bit of $\gamma_{b}=5 \mathrm{~dB}$ over multipath Rayleigh-fading channels.

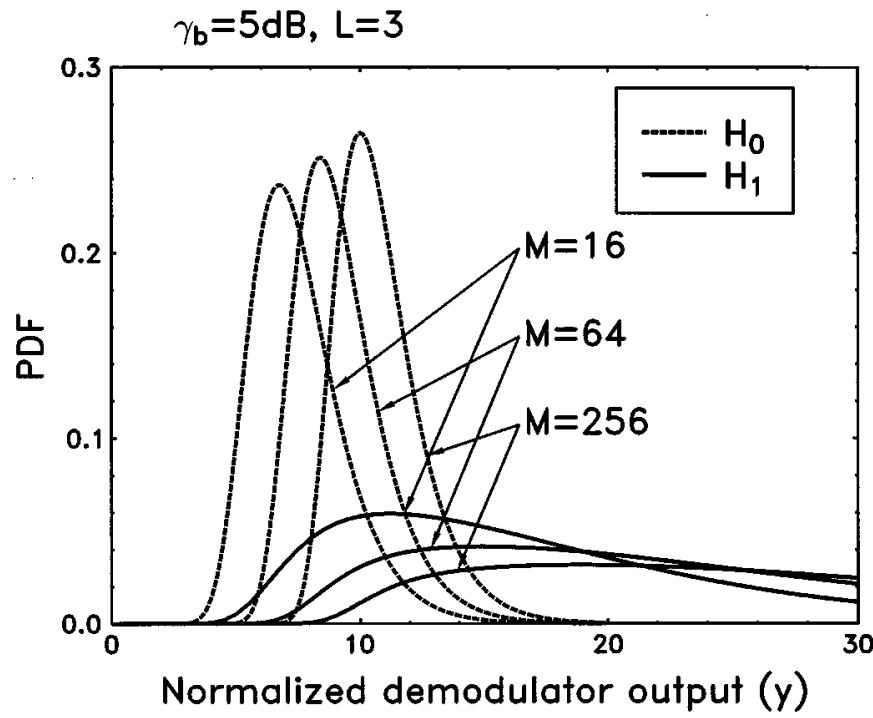

Fig. 3. RTT: PDF of $\lambda={ }^{1} \max \{\cdot\}^{2} \max \{\cdot\}$ according to (18) and (20) under the hypotheses of $H_{1}$ and $H_{0}$ using $\gamma_{b}=0,10,20 \mathrm{~dB}, L=3, M=256$ over multipath Rayleigh-fading channels.

become higher, when increasing the value of $M$, while the distributions of $f_{\lambda}\left(y \mid H_{1}\right)$ for different values of $M$ are very close to each other.

The efficiency of Viterbi's RTT in (5) depends on the distributions of $f_{\lambda}\left(y \mid H_{1}\right)$ and $f_{\lambda}\left(y \mid H_{0}\right)$, specifically, on the overlapping area between these distributions. Usually, in conjunction with the RTT, an erasure is inserted if the RTTs output variable $\lambda$ is lower than a preset threshold, which has to be optimized. How we compute the optimum threshold mathematically, in order to minimize the codeword decoding error probability, remains to be resolved. Hence, the optimum ratio threshold for the RTT can only be found by numerical computation or simulation. Fortunately, for Viterbi's RTT, according to the distributions of $f_{\lambda}\left(y \mid H_{1}\right)$ and $f_{\lambda}\left(y \mid H_{0}\right)$ in Figs. 2 and 3 as well as the other related results in Section $\mathrm{V}$, the optimum threshold $\lambda$ is typically fairly constant and is associated with a value slightly higher than one.

Let $\lambda_{T}$ be a preset threshold invoked in order to erase the low-reliability RS code symbols. Then, for the RTT, $P_{c}, P_{t}$ can be computed with the aid of (1) and (2) as follows:

$$
\begin{aligned}
& P_{c}=P\left(H_{1}\right) \cdot \int_{\lambda_{T}}^{\infty} f_{\lambda}\left(y \mid H_{1}\right) d y \\
& P_{t}=P\left(H_{0}\right) \cdot \int_{\lambda_{T}}^{\infty} f_{\lambda}\left(y \mid H_{0}\right) d y
\end{aligned}
$$

and the erasure probability $P_{e}$ can be computed from (4). Finally, the codeword decoding error probability, $P_{E}$, can be computed by substituting $P_{t}$ and $P_{e}$ into (9) or (10).

\section{ERASURE INSERTION USING THE DEMOdULATION OUTPUT THRESHOLD TEST}

The distributions of the maximum of the decision variables, i.e., the demodulator's output using MLD, under the hypotheses of $H_{1}$ and $H_{0}$, also exhibit distinguishable characteristics and hence can be used in making erasure insertion decisions. Explicitly, we can opt for deciding upon $H_{1}$ or $H_{0}$ based on the observation of the demodulator's output statistics, i.e., erasures can be inserted based on the OTT of the demodulator's output statistics. This claim will be supported by experimental evidence during our further discourse.

Let the demodulator's output be denoted by $Y$, which is defined in (6). Then the distributions of $Y$ under the hypotheses $H_{1}$ of correct detection and $H_{0}$ of erroneous detection are given by (32) and (37) (see the Appendix for the derivation), which are repeated here for convenience

$$
\begin{aligned}
f_{Y}\left(y \mid H_{1}\right)= & \frac{1}{P\left(H_{1}\right)} \cdot \frac{1}{\left(1+\bar{\gamma}_{0}\right)^{L}(L-1) !} y^{L-1} \\
& \cdot \exp \left(-\frac{y}{1+\bar{\gamma}_{0}}\right)[1-\Psi(y)]^{M-1}, \quad y \geq 0 \\
f_{Y}\left(y \mid H_{0}\right)= & \frac{1}{P\left(H_{0}\right)} \cdot \frac{M-1}{(L-1) !} y^{L-1} \exp (-y) \\
& \cdot[1-\Psi(y)]^{M-2}\left[1-\Psi\left(\frac{y}{1+\bar{\gamma}_{0}}\right)\right], \quad y \geq 0
\end{aligned}
$$

where $P\left(H_{1}\right)$ and $P\left(H_{0}\right)$ represent the correct and erroneous detection probabilities, or the a priori probabilities of the second-stage detection of $H_{1}$ and $H_{0}$, while $\Psi(y)$ is given by (21).

The distributions of $f_{Y}\left(y \mid H_{1}\right)$ and $f_{Y}\left(y \mid H_{0}\right)$ are shown in Figs. 4 and 5 for the same parameters as in Figs. 2 and 3, respectively. From Fig. 5 we observe that, for a given value of $M$, the distribution peak of $f_{Y}\left(y \mid H_{0}\right)$ will shift slightly to the right-hand side, when increasing the SNR per bit. However, the peak of $f_{Y}\left(y \mid H_{0}\right)$ will be around a constant value of $y$. In the example of Fig. 5, associated with $M=256$, the peak is located at $y \approx 10$. In other words, this value is usually higher than $y=1$, which represents a typical value characterized by 


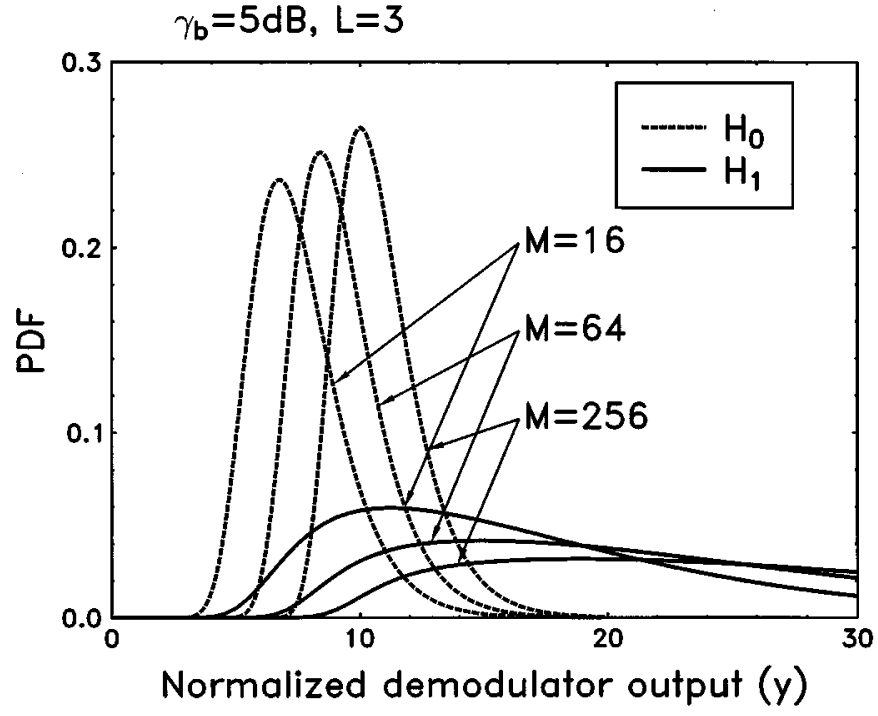

Fig. 4. OTT: The PDF of $Y=\max \{\cdot\}$ according to (25) and (26) under the hypotheses of $H_{1}$ and $H_{0}$ using $M=32,64,128,256, L=3, \gamma_{b}=5 \mathrm{~dB}$ over multipath Rayleigh-fading channels.

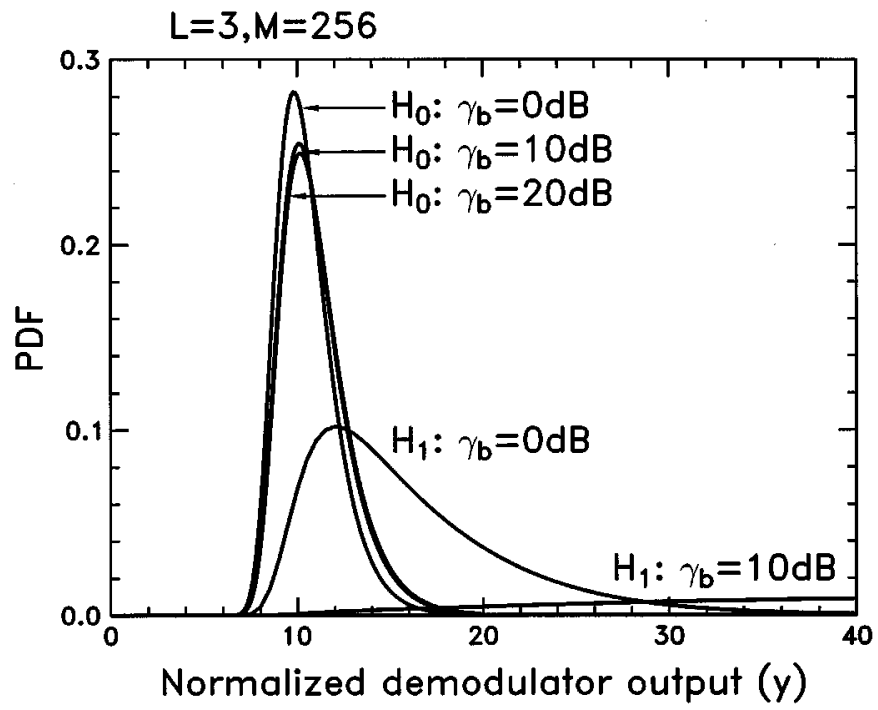

Fig. 5. OTT: The PDF of $Y=\max \{\cdot\}$ according to (25) and (26) under the hypotheses of $H_{1}$ and $H_{0}$ using $\gamma_{b}=0,10,20 \mathrm{~dB}, L=3, M=256$ over multipath Rayleigh-fading channels.

$f_{\lambda}\left(y \mid H_{0}\right)$ of the RTT, as shown in Figs. 2 and 3. For a given value $\gamma_{b}$ of SNR per bit and for different values of $M$, the distributions of $f_{Y}\left(y \mid H_{0}\right)$ and $f_{Y}\left(y \mid H_{1}\right)$ associated with the OTT are significantly different, and the distribution peaks will significantly shift to the right-hand side, when increasing the value of $M$. Furthermore, upon comparing Fig. 3 to Fig. 5 associated with $\gamma_{b}=0 \mathrm{~dB}$ and $10 \mathrm{~dB}$, we find that the distributions of $f_{\lambda}\left(y \mid H_{1}\right)$ and $f_{\lambda}\left(y \mid H_{0}\right)$ for the RTT are more overlapped with each other than the distributions of $f_{Y}\left(y \mid H_{1}\right)$ and $f_{Y}\left(y \mid H_{0}\right)$ for the OTT. As we discussed previously, the efficiency of an erasure insertion scheme depends on the separability of the distributions under the hypotheses of $H_{1}$ and $H_{0}$. Therefore, for a given SNR value and a given $M$ value, the codeword decoding error probability of the OTT will be lower than that of the RTT, as shown in Figs. 8-11.

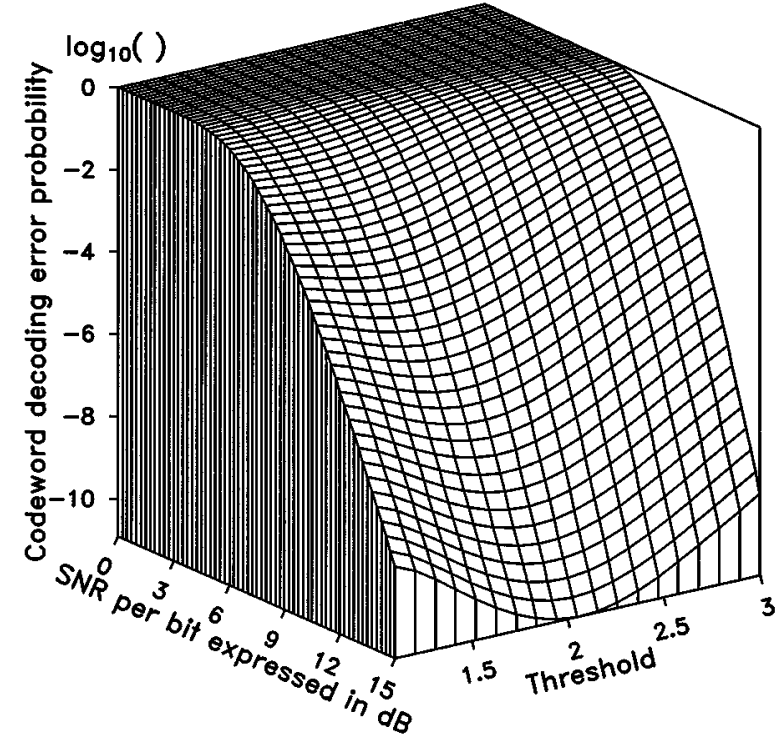

Fig. 6. RTT: codeword decoding error probability versus the SNR per bit, $\gamma_{b}$ and the threshold, $\lambda_{T}$ for the erasure insertion scheme of RTT computed from (23), (24), and (9) using parameters of $L=2, M=32$ and the $\operatorname{RS}(32,20)$, GF(32) code over multipath Rayleigh fading channels.

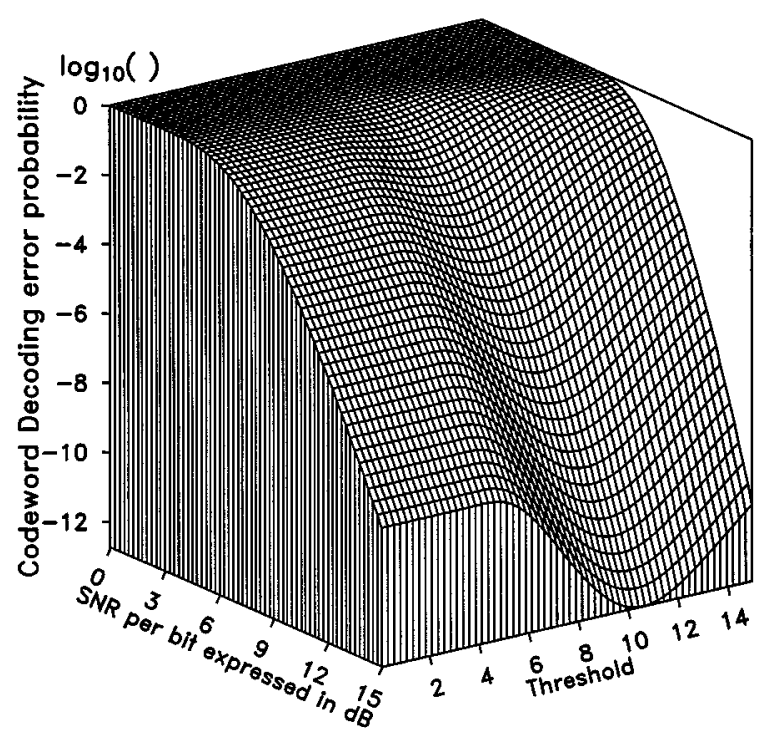

Fig. 7. OTT: codeword decoding error probability versus the SNR per bit, $\gamma_{b}$ and the threshold, $Y_{T}$ for the erasure insertion scheme of OTT computed from (27), (28), and (9) using parameters of $L=2, M=32$ and the $\operatorname{RS}(32,20)$, GF(32) code over multipath Rayleigh fading channels.

Let $Y_{T}$ be a threshold associated with making an erasure decision based on the OTT. Then the correct symbol probability, $P_{c}$, and symbol error probability, $P_{t}$, after erasure insertion but before FEC decoding can be expressed as

$$
\begin{aligned}
& P_{c}=P\left(H_{1}\right) \cdot \int_{Y_{T}}^{\infty} f_{Y}\left(y \mid H_{1}\right) d y \\
& P_{t}=P\left(H_{0}\right) \cdot \int_{Y_{T}}^{\infty} f_{Y}\left(y \mid H_{0}\right) d y
\end{aligned}
$$

and the erasure probability $P_{e}$ can be computed from (4). Finally, the codeword decoding error probability, $P_{E}$, after "errors-and-erasures" decoding can be computed by substituting $P_{t}, P_{e}$ into (9) or (10). 


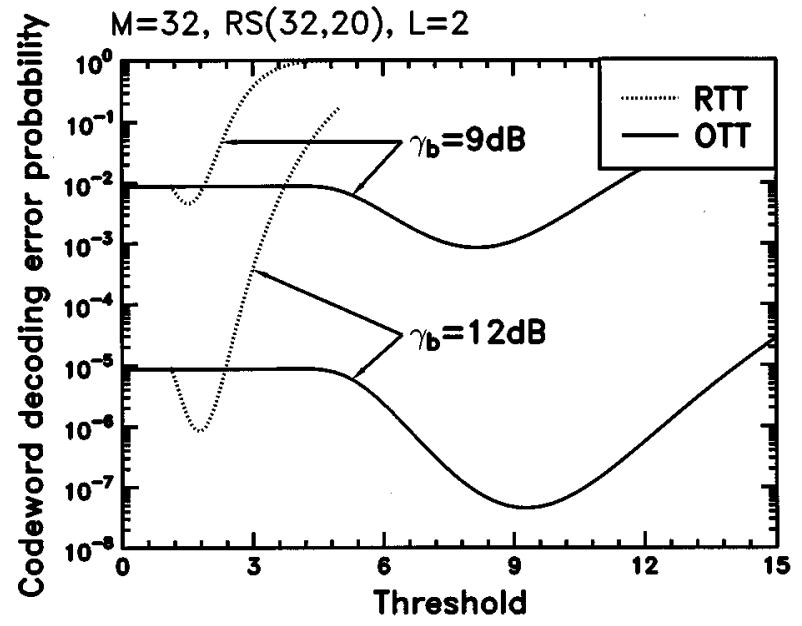

Fig. 8. Codeword decoding error probability versus the threshold for the $\operatorname{RS}(32,20)$ code using 'errors-and-erasures' decoding with parameters of $M=n=32, k=20, L=2$ and $\gamma_{b}=9 \mathrm{~dB}, 12 \mathrm{~dB}$ over multipath Rayleigh fading channels.

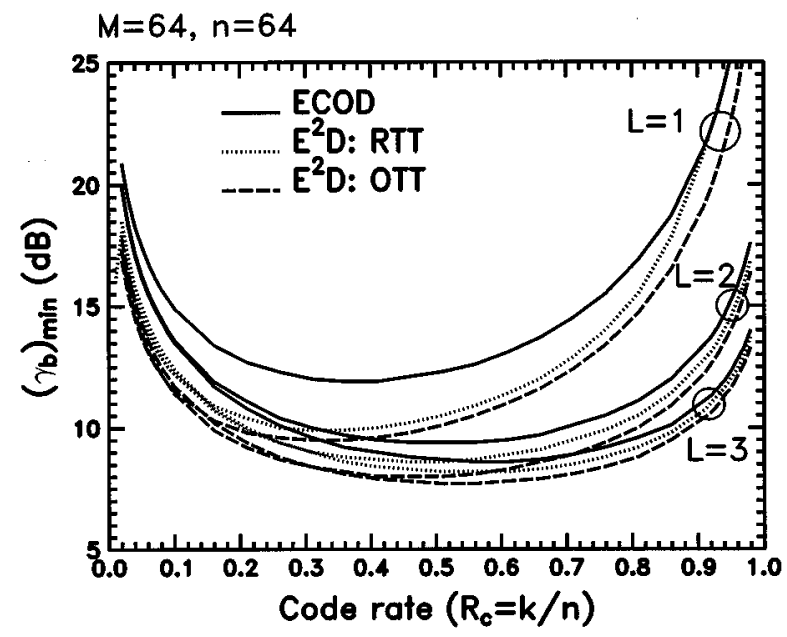

Fig. 9. Minimum SNR per bit, $\gamma_{b}$, required to achieve the codeword decoding error probability of $1 \times 10^{-6}$ in (10) versus RS code rate $R_{c}=k / n$ performance comparison between "error-correction only" decoding (ECOD) and "errors-and-erasures" decoding $\left(E^{2} D\right)$ using the parameters of $M=n=64$ and $L=1,2,3$ over multipath Rayleigh fading channels.

\section{NUMERICAL RESULTS AND DISCUSSION}

In this section, the performance of RS and RRNS codes using "errors-and-erasures" decoding is estimated and compared to that of using "error-correction-only" decoding.

As an example, Figs. 6 and 7 show the codeword decoding error probability of (9) over multipath Rayleigh fading channels for the $\mathrm{RS}(32,20)$ code over the Galois field $\mathrm{GF}(32)=\mathrm{GF}\left(2^{5}\right)$ corresponding to 5-bit symbols using "errors-and-erasures" decoding. In the computations for Fig. 6, erasures were inserted according to Viterbi's RTT scheme, while in Fig. 7, erasures were introduced according to the OTT scheme. In these figures, the codeword decoding error probabilities were computed for different values of SNR per bit and for different thresholds, in order to find the optimum thresholds for both erasure schemes. From the results we observe that for a constant SNR per bit, $\gamma_{b}$ and for both erasure insertion schemes, there exists an optimum threshold, for which the "errors-and-erasures" decoding achieves the minimum codeword decoding error prob-

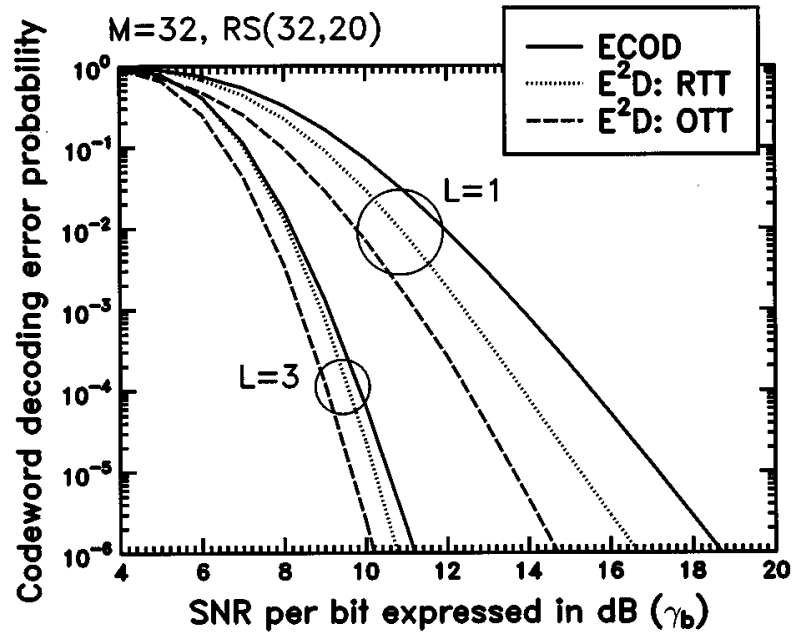

Fig. 10. Codeword decoding error probability versus SNR per bit, $\gamma_{b}$ for the $\operatorname{RS}(32,20)$ code using "error-correction-only" decoding $(E C O D)$ and "errors-and-erasures" decoding $\left(E^{2} D\right)$ with parameters of $M=n=32, k=20$ and $L=1,3$ over multipath Rayleigh fading channels.

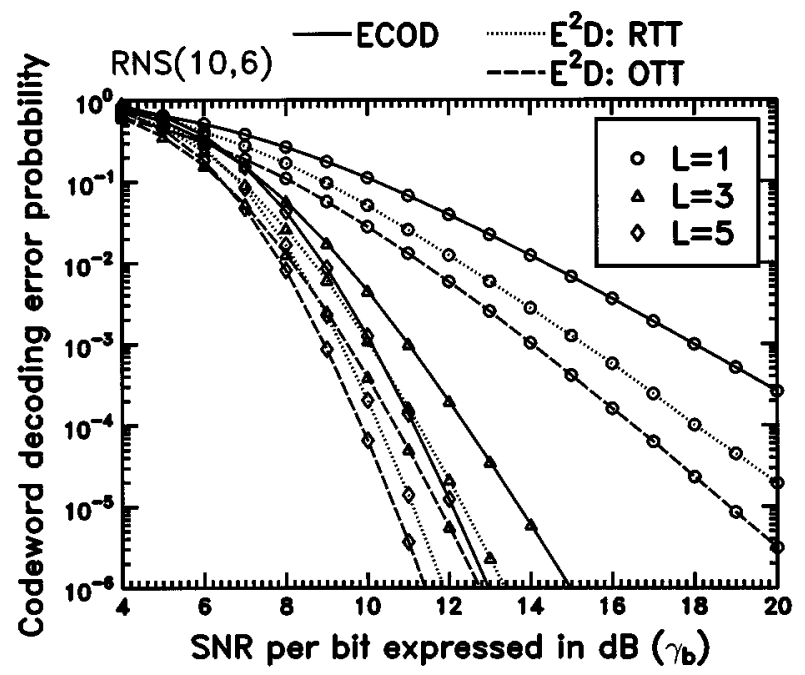

Fig. 11. Codeword decoding error probability versus SNR per bit, $\gamma_{b}$ for the RNS $(10,6)$ code using error-correction-only decoding (ECOD) and "errors-and-erasures" decoding $\left(E^{2} D\right)$ with diversity orders of $L=1,3,5$ and moduli values of $\{121,123,125,127,128,129,131,133,137,139\}$ over multipath Rayleigh fading channels.

ability. Hence, an inappropriate threshold may lead to much higher codeword decoding error probability than the minimum seen in the figures. Observe furthermore that for the erasure insertion scheme using Viterbi's RTT, the optimum threshold assumes values around 1.5 to 2.0 , even though the SNR per bit changes over a large dynamic range from about 6 to $15 \mathrm{~dB}$. By contrast, for the erasure insertion scheme using the OTT, the optimum thresholds are subject to more variations as a function of the SNR per bit than the erasure insertion scheme using the RTT. As shown in Fig. 7, the optimum thresholds range from 6 to 11, when the SNR per bit changes from 6 to $15 \mathrm{~dB}$. However, when using the optimum threshold for any given SNR per bit value, the codeword decoding error probability of the OTT becomes lower than that of the RTT.

The above observation can be further augmented with the aid of Fig. 8, where the codeword decoding error probability versus 
the threshold associated with both the RTT and OTT was estimated and compared using the parameters of $M=32$ and $\gamma_{b}=9 \mathrm{~dB}, 12 \mathrm{~dB}$ for the $\mathrm{RS}(32,20)$ code. The optimum thresholds for the RTT were 1.5 and 1.8, while for the OTT were 8.1 and 9.35 at the SNR per bit values of $9 \mathrm{~dB}$ and $12 \mathrm{~dB}$, respectively. It can be shown that for the RTT the variation of the optimum threshold is only 0.3 , while for the OTT the corresponding variation of the optimum threshold is 1.25 , when the SNR per bit increases from $9 \mathrm{~dB}$ to $12 \mathrm{~dB}$. However, when the optimum thresholds were used, the codeword decoding error probability of the OTT scheme was lower than that of the RTT for both $\gamma_{b}=9 \mathrm{~dB}$ and $12 \mathrm{~dB}$.

In Fig. 9 we estimated the minimum SNR per bit required for achieving the codeword decoding error probability of $1 \times 10^{-6}$, when using (10), for a given code rate $R_{c}=k / n$. The required SNR per bit was computed versus the code rate, $R_{c}$, for the diversity orders of $L=1,2,3$ and for the 64 symbol long $\mathrm{RS}$ code family of $\mathrm{RS}(64, k)$ over the Galois field $\mathrm{GF}(64)=\mathrm{GF}\left(2^{6}\right)$ using "error-correction-only" decoding and "errors-and-erasures" decoding employing both the RTT erasure insertion scheme and the OTT scheme, respectively. The results imply that, for all of the decoding schemes, the optimum code rate, i.e., the code rate that can achieve the required codeword decoding error probability with the lowest SNR per bit, increases when increasing the order of the diversity combining capability. For example, for 64-length RS codes using "error-correction-only" decoding, the optimum code rate for $L=1$ is about 0.4 , for $L=2$ is about 0.6 , while for $L=3$ it is somewhat higher than that for $L=2$. The results also show that, for any given code rate, the minimum required SNR per bit for the "error-correction-only" decoding in order to achieve the target codeword decoding error probability is higher than that for "errors-and-erasures" decoding. Furthermore, the results of Figs. 6-11 indicate that, for a given SNR per bit, in the case of optimum threshold setting for both RTT and OTT, the OTT outperforms the RTT.

In Figs. 10 and 11, the codeword decoding error probability performance of two specific codes was evaluated against the SNR per bit. The $\mathrm{RS}(32,20)$ code over $\mathrm{GF}(32)=\mathrm{GF}\left(2^{5}\right)$ was studied in Fig. 10, while the RRNS $(10,6)$ code is in Fig. 11 with ten moduli taking values of $\{121,123,125,127,128,129$, $131,133,137,139\}$, all of which are close to 128 . This code can correct a maximum of $t=(10-6) / 2=2$ symbol errors or correct a maximum of $\beta=(10-6)=4$ symbol erasures. The first six moduli were the nonredundant moduli, while the last four moduli were the redundant ones. From the results, we observe that under frequency-selective Rayleigh fading, for a constant SNR per bit, for a constant number of diversity components, and also under the assumption that the receiver invoked the optimum threshold, the OTT erasure insertion scheme outperforms the RTT scheme. We note furthermore that the performance of identical-length $\operatorname{RS}(n, k)$ and $\operatorname{RRNS}(n, k)$ codes using the same number of bits per symbol is similar-provided that they both exist - since they are both maximum distance separable codes.

\section{CONCLUSION}

In this paper, the performance of RS or RRNS coded $M$-ary orthogonal signaling schemes using "errors-and-erasures" decoding has been investigated and compared to that using "error-correction-only" decoding. The transmitted signals were subjected to frequency-selective Rayleigh-fading. Two simplest erasure insertion schemes, namely the RTT and the OTT, have been proposed for quantifying the quality of the communication channels. The probability density functions associated with the RTT and those with the OTT under the hypotheses of correct detection and erroneous detection have been derived and their properties have been analyzed. The numerical results showed that the optimum threshold for the RTT over frequency-selective Rayleigh-fading channels was in the range of $[1.5,2]$, while for the OTT, the optimum threshold was subject to more variation as a function of the average SNR per bit. The numerical results also suggested that over frequency-selective Rayleigh-fading channels, the erasure insertion scheme using the OTT outperforms that using the RTT, provided that both schemes invoked the optimum thresholds. The analysis in this paper can be readily extended to direct-sequence code-division multiple-access (DS-CDMA) wireless communication systems [26] using $M$-ary orthogonal signaling and square-law detection. In our future work we will investigate invoking the proposed channel quality measures in order to control the choice of $M$ in burst-by-burst adaptive duplex transceivers, which can be reconfigured as a function of the instantaneous channel quality in their effort to maintain the required transmission integrity.

\section{APPENDIX I}

THE PDFs OF THE FIRST MAXIMUM, THE "SECOND" MAXIMUM, AND THEIR RATIO UNDER THE HYPOTHESES $H_{1}$ OF CORRECT DETECTION AND $H_{0}$ OF ERRONEOUS DETECTION

In this appendix, we derive the PDFs of the maximum and the "second" maximum of the decision variables, $\left\{U_{1}, U_{2}, \ldots, U_{M}\right\}$ of the demodulator, as well as those of their ratio defined in (5). Both the hypothesis that the demodulator output is correct $\left(H_{1}\right)$ and that it is in error $\left(H_{0}\right)$ are considered, when the channel is modeled as a frequency-selective slow Rayleigh fading medium, where the PDFs of the decision variables are given by (15) and (16).

\section{A. PDFs Under the Hypothesis $H_{1}$}

Let the maximum and the "second" maximum of the decision variables be represented as

$$
\begin{aligned}
& Y_{1}={ }^{1} \max \left\{U_{1}, U_{2}, \ldots, U_{M}\right\} \\
& Y_{2}={ }^{2} \max \left\{U_{1}, U_{2}, \ldots, U_{M}\right\} .
\end{aligned}
$$

Then, the PDF of $Y_{1}$ conditioned on $H_{1}$ can be expressed as

$$
\begin{aligned}
f_{Y_{1}}\left(y \mid H_{1}\right) & =\frac{d}{d y} P\left(Y_{1}<y \mid H_{1}\right) \\
& =\frac{1}{P\left(H_{1}\right)} \cdot \frac{d}{d y} P\left(Y_{1}<y, H_{1}\right) \\
& =\frac{1}{P\left(H_{1}\right)} \cdot \frac{d}{d y} P\left(U_{2}<U_{1}, U_{3}<U_{1}, \ldots,\right. \\
& =\frac{1}{P\left(H_{1}\right)} \cdot \frac{d}{d y} \int_{0}^{y} f_{U_{1}}(x)\left[\int_{0}^{x} f_{U_{2}}(z) d z\right]^{M-1} d x
\end{aligned}
$$


Upon substituting the PDFs of $f_{U_{1}}(x)$ and $f_{U_{2}}(z)$ from (15) and (16) into the above equation, we can simplify it as follows:

$$
\begin{aligned}
f_{Y_{1}}\left(y \mid H_{1}\right)= & \frac{1}{P\left(H_{1}\right)} \cdot \frac{1}{\left(1+\bar{\gamma}_{0}\right)^{L}(L-1) !} y^{L-1} \\
& \cdot \exp \left(-\frac{y}{1+\bar{\gamma}_{0}}\right)[1-\Psi(y)]^{M-1}, \quad y \geq 0
\end{aligned}
$$

where the short-hand $\Psi(y)$ is defined in (21).

Similarly, the PDF of $Y_{2}$ conditioned on $H_{1}$ can be expressed as

$$
\begin{aligned}
f_{Y_{2}}\left(y \mid H_{1}\right)= & \frac{d}{d y} P\left(Y_{2}<y \mid H_{1}\right) \\
= & \frac{1}{P\left(H_{1}\right)} \cdot \frac{d}{d y} P\left(Y_{2}<y, H_{1}\right) \\
= & \frac{M-1}{P\left(H_{1}\right)} \cdot \frac{d}{d y} P\left(U_{1}>U_{i}, U_{2}<U_{i}, U_{3}<U_{i},\right. \\
= & \frac{M-1}{P\left(H_{1}\right)} \cdot \frac{d}{d y} \int_{0}^{y} f_{U_{i}}(x)\left[\int_{x}^{\infty} f_{U_{1}}(z) d z\right] \\
& \cdot\left[\int_{0}^{x} f_{U_{2}}(z) d z\right]^{M-2} d x .
\end{aligned}
$$

Note that, in the third step of (33), we assumed that $U_{i} \neq U_{1}$ is the "second" maximum among the decision variables, i.e., $Y_{2}=U_{i}$. Substituting the PDFs of $U_{i}$ and $U_{2}$ from (16), and the PDF of $U_{1}$ from (15) into (33), we finally arrive at

$$
\begin{aligned}
f_{Y_{2}}\left(y \mid H_{1}\right)= & \frac{1}{P\left(H_{1}\right)} \cdot \frac{M-1}{(L-1) !} y^{L-1} \\
& \cdot \exp (-y)[1-\Psi(y)]^{M-2} \Psi\left(\frac{y}{1+\bar{\gamma}_{0}}\right), \\
& \quad y \geq 0 .
\end{aligned}
$$

After obtaining the PDFs of the maximum and the "second" maximum of the decision variables $\left\{U_{1}, U_{2}, \ldots, U_{M}\right\}$, the PDF of their ratio defined in (5), i.e., $\lambda=Y_{1} / Y_{2}$, conditioned on the maximum being larger than the "second" maximum-i.e., on $Y_{2}<Y_{1}$ - can be derived using the approach of [25, p. 244], which can be derived as

$$
\begin{aligned}
& f_{\lambda}\left(y \mid H_{1}\right) \\
&=\frac{d}{d y} P\left(\lambda=\frac{Y_{1}}{Y_{2}} \leq y \mid Y_{2}<Y_{1}, H_{1}\right) \\
&=\frac{1}{P\left(Y_{2}<Y_{1} \mid H_{1}\right)} \frac{d}{d y} P\left(\lambda=\frac{Y_{1}}{Y_{2}} \leq y, Y_{2}<Y_{1} \mid H_{1}\right) \\
&=\frac{1}{P\left(Y_{2}<Y_{1} \mid H_{1}\right)} \frac{d}{d y} \int_{0}^{\infty} P\left(y_{2}<Y_{1} \leq y_{2} y \mid Y_{2}=y_{2}, H_{1}\right) \\
& \cdot f_{Y_{2}}\left(y_{2} \mid H_{1}\right) d y_{2} \\
&= \frac{1}{P\left(Y_{2}<Y_{1} \mid H_{1}\right)} \frac{d}{d y} \int_{0}^{\infty}\left[\int_{y_{2}}^{y_{2} y} f_{Y_{1}}\left(y_{1} \mid H_{1}\right) d y_{1}\right] \\
& \cdot f_{Y_{2}}\left(y_{2} \mid H_{1}\right) d y_{2} \\
&= \frac{1}{P\left(Y_{2}<Y_{1} \mid H_{1}\right)} \int_{0}^{\infty} y_{2} f_{Y_{1}}\left(y_{2} y \mid H_{1}\right) f_{Y_{2}}\left(y_{2} \mid H_{1}\right) d y_{2}, \\
& y \geq 1
\end{aligned}
$$

where $P\left(Y_{2}<Y_{1} \mid H_{1}\right)$ represents the probability of $Y_{2}<Y_{1}$ conditioned on the correct detection of $H_{1}$, which is quantified by (22).
Finally, the distribution of the ratio $\lambda$ in (5) associated with the RTT under the hypothesis $H_{1}$ of correct detection can be simplified to (19).

\section{B. PDFs Under the Hypothesis $H_{0}$}

Under the hypothesis $H_{0}$, the PDF of $Y_{1}$ can be expressed as

$$
\begin{aligned}
f_{Y_{1}}\left(y \mid H_{0}\right)= & \frac{d}{d y} P\left(Y_{1}<y \mid H_{0}\right) \\
= & \frac{1}{P\left(H_{0}\right)} \cdot \frac{d}{d y} P\left(Y_{1}<y, H_{0}\right) \\
= & \frac{1}{P\left(H_{0}\right)} \cdot \frac{d}{d y} P\left(U_{1} \neq Y_{1}<y\right) \\
= & \frac{M-1}{P\left(H_{0}\right)} \cdot \frac{d}{d y} P\left(U_{1}<U_{i}, U_{2}<U_{i}, \ldots,\right. \\
= & \frac{M-1}{P\left(H_{0}\right)} \cdot \frac{d}{d y} \int_{0}^{y} f_{U_{i}}(x)\left[\int_{0}^{x} f_{U_{1}}(z) d z\right] \\
& \cdot\left[\int_{0}^{x} f_{U_{2}}(z) d z\right]^{M-2} d x .
\end{aligned}
$$

After using the corresponding PDFs of $U_{1}$ and $U_{j}$ for $j \neq 1$, (36) can be simplified as

$$
\begin{aligned}
& f_{Y_{1}}\left(y \mid H_{0}\right)= \frac{1}{P\left(H_{0}\right)} \cdot \frac{M-1}{(L-1) !} y^{L-1} \exp (-y) \\
& \cdot[1-\Psi(y)]^{M-2}\left[1-\Psi\left(\frac{y}{1+\bar{\gamma}_{0}}\right)\right], \\
& y \geq 0 .
\end{aligned}
$$

The PDF of $f_{Y_{2}}\left(y \mid H_{0}\right)$ conditioned on $H_{0}$ can be expressed as

$$
\begin{aligned}
& f_{Y_{2}}\left(y \mid H_{0}\right) \\
&=\frac{d}{d y} P\left(Y_{2}<y \mid H_{0}\right) \\
&=\frac{1}{P\left(H_{0}\right)} \cdot \frac{d}{d y} P\left(Y_{2}<y, H_{0}\right) \\
&=\frac{1}{P\left(H_{0}\right)} \cdot\left[\frac{d}{d y} P\left(U_{1}=Y_{2}<y\right)+\frac{d}{d y} P\left(U_{1} \neq Y_{2}<y\right)\right] \\
&=\frac{M-1}{P\left(H_{0}\right)} \cdot\left\{\frac { d } { d y } P \left(U_{i}>U_{1}, U_{2}<U_{1}, \ldots, U_{M}<U_{1} ;\right.\right. \\
&\left.U_{1}=Y_{2}<y\right)+(M-2) \frac{d}{d y} P\left(U_{i}>U_{j}, U_{1}\right. \\
&\left.\left.<U_{j}, \ldots, U_{M}<U_{j} ; U_{j}=Y_{2}<y\right)\right\} \\
&=\frac{M-1}{P\left(H_{0}\right)} \cdot\left\{\frac{d}{d y} \int_{0}^{y} f_{U_{1}}(x)\left[\int_{x}^{\infty} f_{U_{i}}(z) d z\right]\right. \cdot\left[\int_{0}^{x} f_{U_{2}}(z) d z\right]^{M-2} d x+(M-2) \frac{d}{d y} \\
& \int_{0}^{y} f_{U_{j}}(x)\left[\int_{x}^{\infty} f_{U_{i}}(z) d z\right]\left[\int_{0}^{x} f_{U_{1}}(z) d z\right] \\
&\left.\cdot\left[\int_{0}^{x} f_{U_{2}}(z) d z\right]^{M-3} d x\right\}
\end{aligned}
$$

where at the third step of the derivation, the "second" maximum can be $U_{1}$ or any one of the other decision variables. At the fourth step, we assumed that $U_{i}$ was the maximum, and $U_{j} \neq$ 
$U_{1}, U_{i}$ was the "second" maximum, when it was not $U_{1}$. After substituting the corresponding PDFs in the above equation, we simplify it to

$$
\begin{aligned}
f_{Y_{2}}\left(y \mid H_{0}\right) & \frac{1}{P\left(H_{0}\right)} \cdot \frac{M-1}{(L-1) !} y^{L-1} \Psi(y)[1-\Psi(y)]^{M-3} \\
& \cdot\left\{\frac{1}{\left(1+\bar{\gamma}_{0}\right)^{L}} \exp \left(-\frac{y}{1+\bar{\gamma}_{0}}\right)[1-\Psi(y)]\right. \\
& \left.+(M-2) \exp (-y)\left[1-\Psi\left(\frac{y}{1+\bar{\gamma}_{0}}\right)\right]\right\}, \\
y \geq 0 . &
\end{aligned}
$$

Again, the distribution of the ratio $\lambda$ in (5) associated with the RTT under the hypothesis $H_{0}$ of erroneous demodulation can be simplified to (20) using (35) under the hypothesis of $H_{0}$.

\section{ACKNOWLEDGMENT}

The authors would like to thank A. Svensson, the Editor handling the manuscript, and the anonymous reviewers for their valuable comments and suggestions which enhanced the quality of the paper.

\section{REFERENCES}

[1] R. E. Blahut, Theory and Practice of Error Control Codes. Reading, MA: Addison-Wesley, 1983.

[2] G. D. Forney, "Exponential error bounds for erasure, list, and decision feedback scheme," IEEE Trans. Inform. Theory, vol. IT-14, pp. 206-220, Mar. 1968.

[3] A. J. Viterbi, "A robust ratio-threshold technique to mitigate tone and partial band jamming in coded MFSK systems," in Proc. IEEE Military Commun. Conf. Rec., Oct. 1982, pp. 22.4.1-22.4.5.

[4] L. F. Chang and R. J. McEliece, "A study of Viterbi's ratio threshold AJ technique," in Proc. IEEE Military Commun. Conf. Rec., Oct. 1984, pp. 11.2.1-11.2.5.

[5] C. W. Baum and M. B. Pursley, "Bayesian methods for erasure insertion in frequency-hop communication system with partial-band interference," IEEE Trans. Commun., vol. 40, pp. 1231-1238, July 1992.

[6] — , "Bayesian generation of dependent erasures for frequency-hop communications and fading channels," IEEE Trans. Commun., vol. 44 pp. 1720-1729, Dec. 1996

[7] S. W. Kim and W. Stark, "Performance limits of Reed-Solomon coded CDMA with orthogonal signaling in a Rayleigh-fading channel," IEEE Trans. Commun., vol. 46, pp. 1125-1134, Sept. 1998.

[8] L. L. Yang, K. Yen, and L. Hanzo, "A Reed-Solomon coded DS-CDMA system using noncoherent $M$-ary orthogonal modulation over multipath fading channels," IEEE J. Select. Areas Commun., vol. 18, pp. 2240-2251, Nov. 2000.

[9] C. M. Keller and M. B. Pursley, "Diversity combining for channels with fading and partial-band interference," IEEE J. Select. Areas Commun., vol. SAC-5, pp. 248-260, Feb. 1987

[10] R. Steele and L. Hanzo, Mobile Radio Communications. New York: Wiley-IEEE Press, 1999, ch. 4.

[11] K. W. Watson, "Self-checking computations using residue arithmetic," Proc. IEEE, vol. 54, pp. 1920-1931, Dec. 1966.

[12] R. J. Cosentino, "Fault-tolerance in a systolic residue arithmetic processor array," IEEE Trans. Computers, vol. 37, pp. 886-890, July 1988.

[13] E. D. D. Claudio, G. Orlandi, and F. Piazza, "A systolic redundant residue arithmetic error correction circuit," IEEE Trans. Computers, vol. 42, pp. 427-432, Apr. 1993.

[14] H. Krishna, K. Y. Lin, and J. D. Sun, "A coding theory approach to error control in redundant residue number system-Part I: Theory and single error correction," IEEE Trans. Circuits Systems, vol. 39, pp. 8-17, Jan. 1992.

[15] J. D. Sun and H. Krishna, "A coding theory approach to error control in redundant residue number system-Part II: Multiple errors detection and correction theory," IEEE Trans. Circuits Systems, vol. 39, pp. 18-32, Jan. 1992.
[16] H. Krishna and J. D. Sun, "On theory and fast algorithms for error correction in residue number system product codes," IEEE Trans. Computers, vol. C-42, pp. 840-852, July 1993.

[17] F. Barsi and P. Maestrini, "Error correcting properties of redundant residue number systems," IEEE Trans. Computers, vol. C-22, pp. 307-317, Mar. 1973.

[18] L. L. Yang and L. Hanzo, "Coding theory and performance of redundant residue number system codes," IEEE Trans. Inform. Theory, Aug. 1999, submitted for publication.

[19] — - "Performance of residue number system based DS-CDMA over multipath fading channels using orthogonal sequences," Euro. Trans. Telecommun., vol. 9, no. 6, pp. 525-536, Nov.-Dec. 1998.

[20] — "RNS arithmetic assisted $M$-ary modulation," IEEE Commun. Lett., vol. 3, pp. 28-30, Jan. 1999.

[21] S. M. Kay, Fundamentals of Statistical Signal Processing: Detection Theory. Englewood Cliffs, NJ: Prentice-Hall, 1993.

[22] A. D. Whalen, Detection of Signals in Noise. New York: Academic, 1971.

[23] EIA/TIA-95 Rev A, "Mobile station-base station compatibility standard for dual-mode wideband spread spectrum cellular system,", 1995.

[24] J. G. Proakis, Digital Communications, 3rd ed. New York: McGrawHill, 1995.

[25] H. J. Larson and B. O. Shubert, Probabilistic Models in Engineering Sciences, Volume I: Random Variables and Stochastic Processes. New York: Wiley, 1979.

[26] L. M. A. Jalloul and J. M. Holtzman, "Performance analysis of DS/CDMA with noncoherent $M$-ary orthogonal modulation in multipath fading channels," IEEE J. Select. Areas Commum., vol. 12, pp. 862-870, June 1994.

Lie-Liang Yang (M'98) received the B.Eng. degree in communication engineering from Shanghai TieDao University, Shanghai, China, in 1988, and the M.S and Ph.D. degrees in communications and electronics from Northern Jiaotong University, Beijing, China, in 1991 and 1997, respectively.

From 1991 to 1993, he was a Lecturer with the Department of Electrical Engineering, East-China Jiaotong University, China. From 1993 to 1997, he was with the Modern Communications Research Institute, Northern Jiaotong University, China. From June 1997 to December 1997, he was a Visiting Scientist of the Institute of Radio Engineering and Electronics, Academy of Sciences of the Czech Republic. Since December 1997, he has been with the Communication Group, Department of Electronics and Computer Science, University of Southampton, U.K. and has been involved in researching various error correction coding, modulation schemes, synchronization and CDMA systems for future generations of wireless mobile communication systems. He has published about 50 papers in journals and conference proceedings.

Dr. Yang was awarded the Royal Society Sino-British Fellowship in 1997.

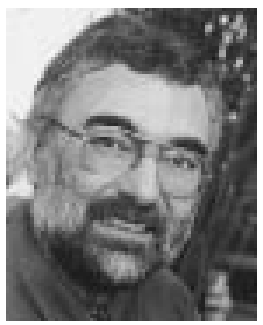

Lajos Hanzo (SM'92) received the Dipl. Ing. degree in electronics in 1976 and the Ph.D. degree in 1983 , both from the Technical University of Budapest, Hungary

During his 24-year career in telecommunications, he has held various research and academic posts in Hungary, Germany, and the U.K. Since 1986, he has been with the Department of Electronics and Computer Science, University of Southampton, Southampton, U.K., and has been a Consultant to Multiple Access Communications Ltd. Southampton, U.K. Currently he holds a chair in telecommunications. He co-authored five books on mobile radio communications, published about 300 research papers, organized and chaired conference sessions, presented overview lectures, and was awarded a number of distinctions. Currently he is managing an academic research team, working on a range of research projects in the field of wireless multimedia communications sponsored both by industry and under the auspices of the Engineering and Physical Sciences Research Council (EPSRC), U.K., the European IST Programme, and the Mobile Virtual Centre of Excellence (VCE), U.K. For further information on research in progress and associated publications, please refer to http://www-mobile.ecs.soton.ac.uk. 